\title{
Primary Alveolar Rhabdomyosarcoma of Breast: - A Rare Case Report
}

\author{
Dr. Rachna Kumari ${ }^{1,}$ Dr. Suryajit Kumar Singh ${ }^{2,}$ Dr Ajeet Kumar ${ }^{3,}$ \\ Dr Amit Kumar Sinha ${ }^{4}$ Dr Anju Singh ${ }^{5}$ \\ ${ }^{I} P G$ Student ,Department Of Pathology, IMS \&SUM Hospital Bhubaneshwar \\ ${ }^{2}$ Senior Resident IGIMS Patna, ${ }^{3}$ Senior Resident IGIMS Patna,${ }^{4}$ Senior Resident IGIMS Patna \\ ${ }^{5}$ Associate Prof, Department Of Pathology IGIMS Patna
}

\begin{abstract}
Sarcoma is a rare malignant tumour of breast. Primary alveolar rhabdomyoarcoma is extremely rare in breast with limited case reports in literature. We report a case of primary alveolar rhabdomyosarcoma of breast in an adolescent girl who presented with progressively increasing firm to hard swelling in the left breast beneath areola. MRI and FNAC was suggestive of lobular carcinoma of breast. Patient underwent lumpectomy with $2 \mathrm{~cm}$ margin and latismus dorsi flap reconstruction. After histopathological examination and immunohistochemistry tumour was diagnosed as alveolar rhabdomyosarcoma and patient is in complete remission after one year of treatment.
\end{abstract}

Keywords: Alveolar rhabdomyosarcoma,Breast, Adolescent

\section{Introduction}

Rhabdomyosarcoma is commonest pediatric soft tissue tumour and rarely occurs in adult. It commonly involves head and neck region, extremities and genitourinary tract. Primary rhabdomyosarcoma arising from breast is less common than metastatic rhabdomyosarcoma. we report here one such case of primary alveolar rhabdomyosarcoma of breast which was mimicking lobular carcinoma of breast on cytology and contrast enhanced Magnetic resonance imaging (CEMRI).

\section{Case history}

A 16 yrs female presented to us with progressive swelling of left breast for 2 months. Clinical examination revealed a $4 \times 4 \mathrm{~cm}$ hard mobile lump in central quadrant of left breast with no abnormality in the right breast. Chest wall and axilla were free with no axillary lymph node enlargement on either side. Mammography showed a high dense mass (? ca breast) in the retroareolar region of left breast. USG also revealed lobulated hypoechoic mass involving nipple. MRI of left breast was suggestive of lobular carcinoma with normal study of right breast. FNAC from the breast lesion was done and it showed tumour cells arranged mostly diffusely, in small clusters and single file suggestive of lobular carcinoma of breast ( fig -1). Further patient underwent lumpectomy with $2 \mathrm{~cm}$ margin and latismus dorsi flap reconstruction. Specimen was sent for histopathological examination. We received lumpectomy specimen measuring $12 \times 10 \times 4 \mathrm{~cm}$ with a well delineated grey tan solid growth measuring $4 \times 3.5 \times 2 \mathrm{~cm}$ (fig - 2). On microscopic examination it revealed round to oval cells with scanty cytoplasm, indistinct cell border, pleomorphic hyperchromatic nuclei arranged in cords, nests and alveolar pattern with fibrous septae(fig - 3). There were frequent mitoses ( 25 mitoses/hpf).A diagnosis of malignant round cell tumour was made and immunohistochemistry was advised for confirmation. Immunohistochemistry was done and it was strongly positive for vimentine (fig -4) and myogenin and diffusely positive for desmin. Tumour was negative for synaptophysin and CD99 thus excluding other malignant round cell tumour and confirming the diagnosis of alveolar rhabdomyosarcoma. The patient was discharged and advised for post operative chemotherapy.

\section{Discussion}

Alveolar rhabdomyosarcoma is one of the two main subtypes of rhabdomyosarcoma family of soft tissue tumour. These are mesenchyal tumours related to the skeletal muscle lineage. The other major subtype is embryonal rhabdomyosarcoma which commonly involves head and neck region $f$ children. Alveolar rhabdomyosarcoma generally occurs in skeletal muscles of extremities. Rhabdomyosarcoma is diagnosed by detection of cross striations characteristic of skeletal muscle under light microscope. Diagnosis is confirmed by immunohistochemistry for Actin, Desmin, Myogenin and myo D1. Immunostaining for myogenin and myo D1 show different patterns between Alveolar rhabdomyosarcoma and Embryonal rhabdomyosarcoma. Tumour cells in alveolar rhabdomyosarcoma are relatively small with scant cytoplasm and round regular nuclei . Botryoid variety is seen in genitourinary tract of young children. Totally resected tumours have better prognosis while 
metastatic disease and residual disease has got bad prognosis . Cells of origin of rhabdomyosarcoma is still debated. It is hypothesised that Myogenic rhabdomyosarcoma arises from satellite cells while Non myogenic RMS arise from mesenchymal progenitor cells. Such cells may circulated in different organs and may give rise to rhabdomyosarcoma.

\section{Conclusion}

Primary alveolar rhabdomyosarcoma of breast is although rare but should be kept as one of the differential diagnosis in adolescent females. Clinical and histopathological correlation is must before formulating treatment.

\section{Reference}

[1]. Parham DM, Ellison DA, Rhabdomyosarcoma in adults and children: An update. Arch Pathol Lab Med 2006:130:1454-65.

[2]. Merlino G. helman LJ. Rhabdomyosarcoma-Working out the pathways: Oncogene 1999;18;5340-8.

[3]. Evans RW. Rhabdomyosarcoma of Breast. J Clin pathol. 1953;6:140-144.

[4]. Hay's DM, Donaldson SS, Shimada H,; Primary and metastatic Rhabdomyosarcoma in the breast neoplasms of adolescent females, a report from the inter group rhabdomyosarcoma Study. Med Ped Onco 1997; 29:181-189.

[5]. Hettmers, Wagers AJ: Uncovering the origins of rhabdomyosarcoma. Nature Medicine: 2010;16:171-173.

[6]. Ventura - Holman T, Hahn H, Subauste JS, Maher JF; The FEM1A gene is down regulated in rhabdomyosarcoma. Tumor Biol 2005;26:294-9.

[7]. Rosen PP. Sarcoma: Breast Pathology. Philadelphia, Pa : Lippincott-Raven: 1997:713-4.

[8]. Tavassoli FA.; Mesenchymal lesions; Pathology of the breast. East Norwalk. Conn: Appleton and Lang: 1999: 706-09

\section{Legends}

Fig. 1 : Photograph of FNAC showing tumour cells arranged mostly diffusely, in small clusters and single file

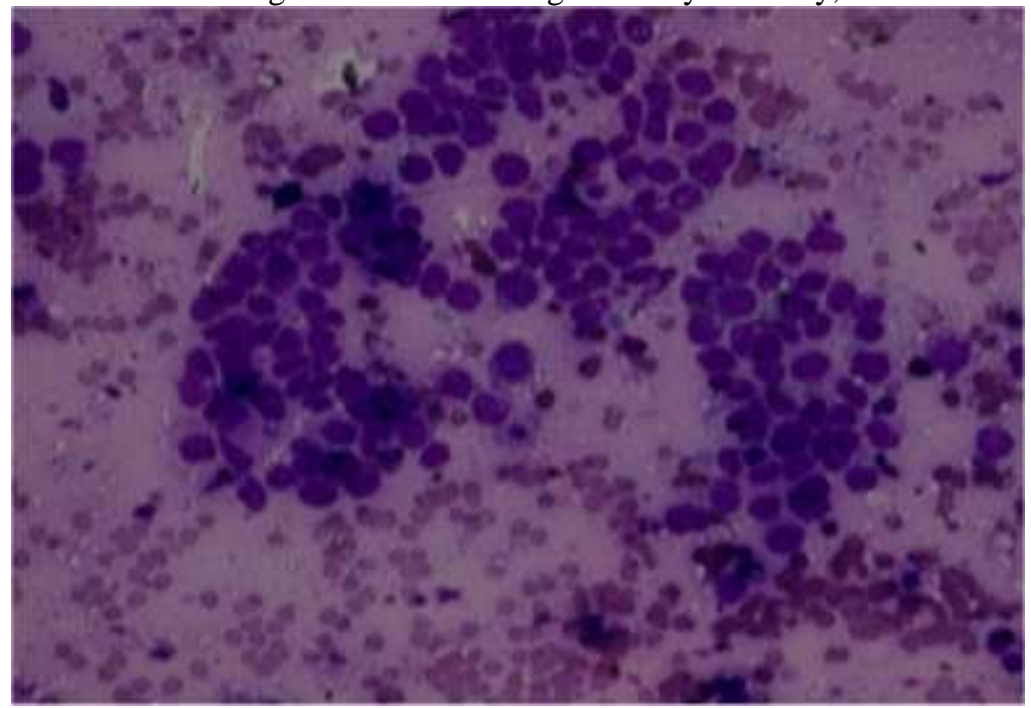

Fig 2: Photograph showing lumpectomy specimen with a well delineated grey tan slid area

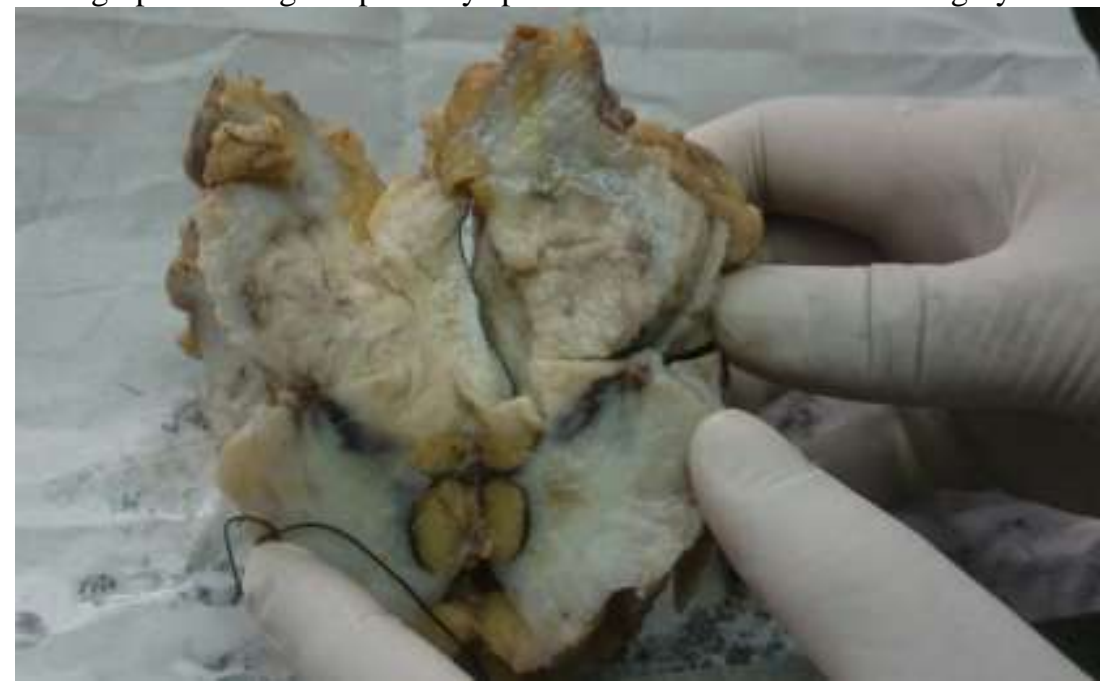


Fig 3: : Photograph showing round to oval cells with scanty cytoplasm, indistinct cell border, pleomorphic hyperchromatic nuclei arranged in cords, nests and alveolar pattern with fibrous septa(H E 400X)

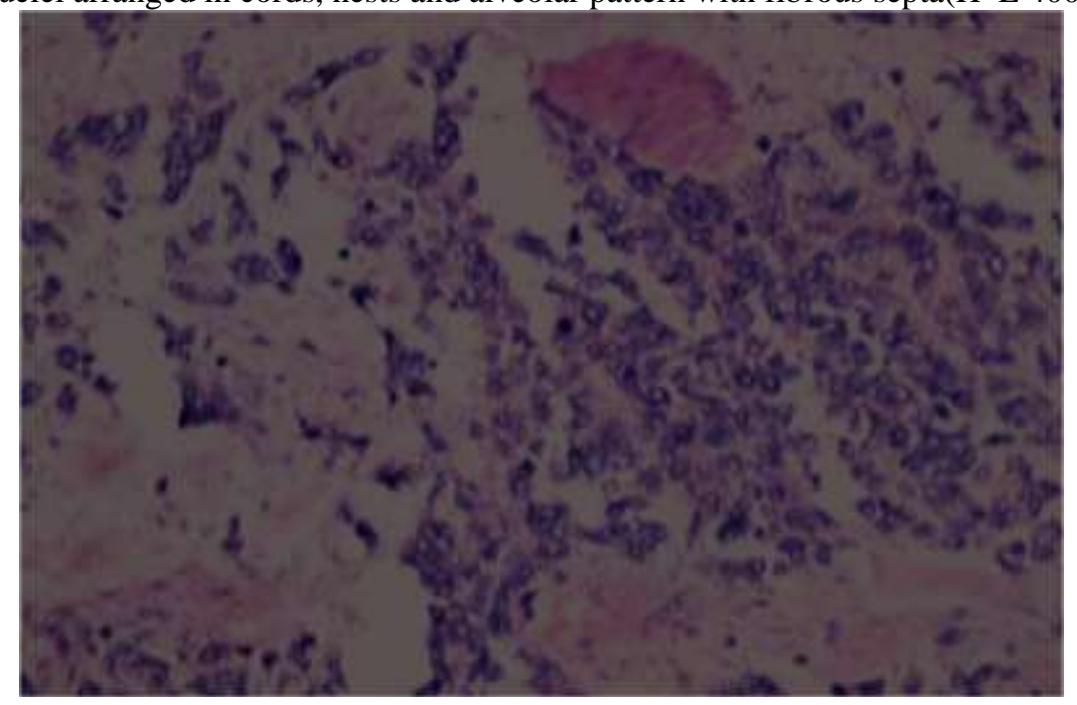

Fig 4: photograph showing tumour cells strongly positive for vimentine

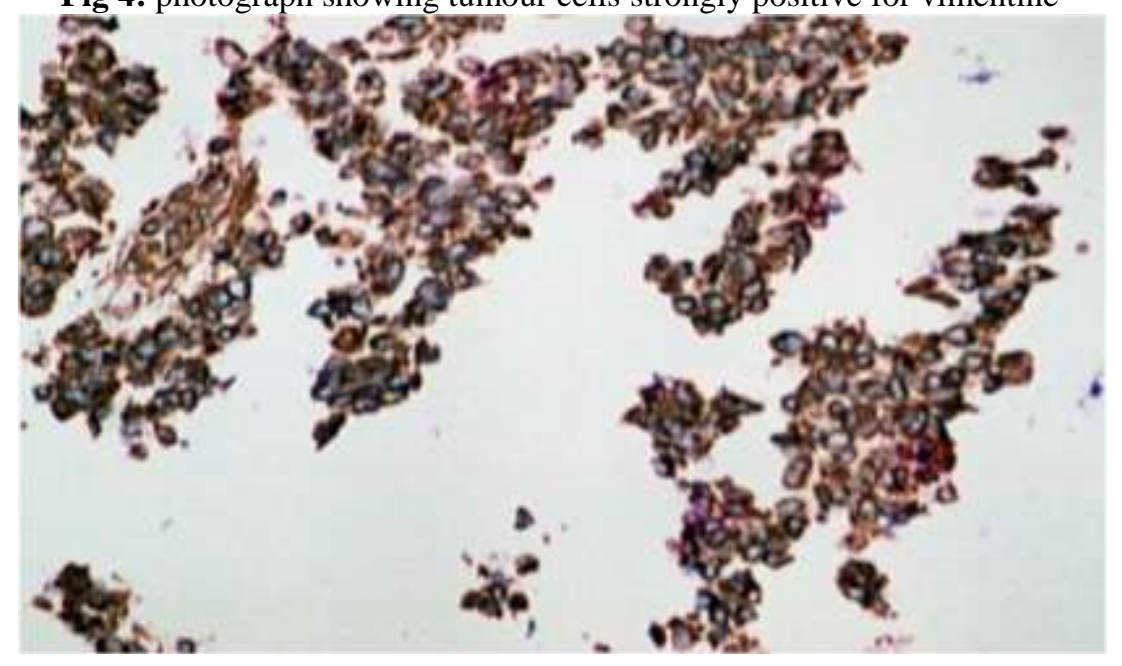

